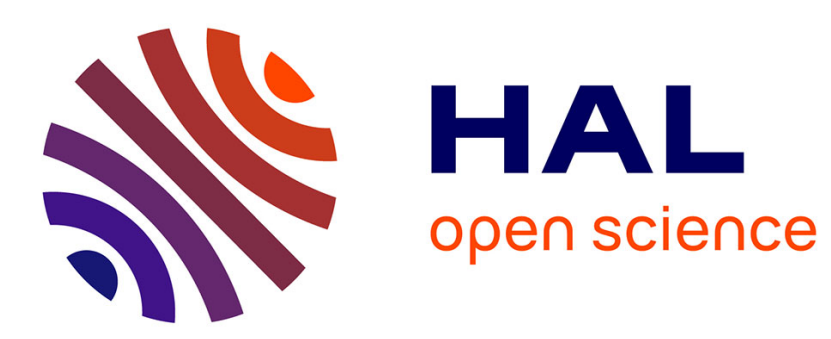

\title{
Localized instability of a granular layer submitted to an ascending liquid flow
}

Patrick Rigord, Alessio Guarino, Valérie Vidal, Jean-Christophe Géminard

\section{To cite this version:}

Patrick Rigord, Alessio Guarino, Valérie Vidal, Jean-Christophe Géminard. Localized instability of a granular layer submitted to an ascending liquid flow. Granular Matter, 2005, 7 (4), pp.191-197. ensl-00180268

\section{HAL Id: ensl-00180268 \\ https://hal-ens-lyon.archives-ouvertes.fr/ensl-00180268}

Submitted on 18 Oct 2007

HAL is a multi-disciplinary open access archive for the deposit and dissemination of scientific research documents, whether they are published or not. The documents may come from teaching and research institutions in France or abroad, or from public or private research centers.
L'archive ouverte pluridisciplinaire HAL, est destinée au dépôt et à la diffusion de documents scientifiques de niveau recherche, publiés ou non, émanant des établissements d'enseignement et de recherche français ou étrangers, des laboratoires publics ou privés. 


\section{Localized instability of a granular layer submitted to an ascending liquid flow}

\begin{abstract}
Using a very simple experimental setup, we study the response of a thin layer of immersed granular material to an ascending liquid-flow; a pressure difference $\Delta P$ is imposed between the two horizontal free surfaces of a thin layer of glass beads, such that the liquid tends to flow upwards, and the resulting flow-rate $v$ is measured. As generally observed in fluidized beds, the layer destabilizes when the pressure force exactly compensates the weight of the grains. At the free surface, one then observes the formation of a localized fountain of granular material the characteristic size of which is found to be proportional to the grain size and, surprizingly, independent of both the flow-rate and the thickness of the granular layer. Simple theoretical arguments account for the main experimental features.
\end{abstract}

Keywords Fluidized beds · Instability · Flow localization

\section{Introduction}

Understanding the mechanical behavior of immersed granular materials is of great practical importance as assemblies of grains immersed in a fluid are involved in many industrial processes (for instance, fluidized beds in which the grains are usually suspended by means of an ascending air-flow [1]) or geophysical situations (for instance, sand ripples, which form

P. Rigord · J.-C. Géminard ( $ه)$

Laboratoire de Physique, Ecole Normale Supérieure de Lyon,

46 Allée d'Italie, 69364 Lyon cedex 07 - France

E-mail: jean-christophe.geminard@ens-lyon.fr

A. Guarino

Université de la Polynésie Française,

B.P. 6570 Faa'a Aéroport, Tahiti - Polynésie Française

V. Vidal

Institut de Physique du Globe,

4, place Jussieu, 75252 Paris cedex 05 - France

V. Vidal · J.-C. Géminard

Nonlinear Physics Laboratory - Universidad de Santiago de Chile, Avenida Ecuador 3493 - Santiago - Chile on the ocean floor, play an important role in the transport of sediments [2]).

Submitted to shear, granular matter has been identified to present friction-like mechanical-properties [3]; the material starts flowing only for a large enough shear-stress and, when the grains are flowing, the shear-stress only slightly depends on the shear-rate. It is important to notice that, in most of the experimental situations, submitted to shear, granular materials only flow within a thin layer, which is usually of about a few grain-size thick [4]. These conclusions hold true even when the grains are immersed in a liquid [5]. The presence of the interstitial fluid (gas or liquid) can lead, in some experimental situations, to puzzling phenomena. For instance, in the case of fine powders, J. Duran observed the formation of droplets from a thin tapped granular-layer [6] and, when placed on an inclined surface, these droplets are shown to climb the slope [7]; these observations can be accounted by the air flow within the grains. Another puzzling example is provided by the sedimentation dynamics of a colloidal gel during which one observes a fast sedimentation process accompanied by a localized destabilization of the upper free-surface [8]; we can consider that, during sedimentation, the assembly of colloidal particles is submitted to an ascending liquid-flow that destabilizes the granular packing. In order to better understand such phenomena, which often involve a localization of the grains motion, it seems relevant to study the effect of a flow of the interstitial fluid within the solid grains.

Interested in the filtering of water, H. Darcy studied the flow of water through a granular packing at rest and established his famous empirical law that relates the flow-rate to the pressure gradient [9]. However, in practice, the response of the granular material can be slightly more complex. For instance, in the case of a deformable granular medium, the flow can induce a permeability anisotropy [10]. These studies apply to cases in which the fluid flow tends to compact the granular packing. To the contrary, a large ascending air- or liquid-flow can be used to fluidize the granular material [1]. Fluidized beds have been widely studied because of their very important engineering applications [11-13]: for 
instance, many industrial devices are used to mix, sort, dry or thermally treat the solid grains. In general, the efficiency of the process depends on the homogeneity of the flow in planes perpendicular to the main upward liquid or gaseous flow that insures the fluidization of the granular material. Thus, most of the devices roughly consist of a vertical cylinder, the height of which is much larger than its diameter. Even if one can distinguish the spouted beds in which the fluid is locally injected at the bottom of the granular column [14], the gas or liquid is generally forced to flow homogeneously from the bottom at a well-controlled constant flow-rate. The different regimes observed when the flow-rate is changed have been widely studied and empirical or theoretical descriptions of the main experimental features are available. However, in order to account for observations in small-size systems (for instance, the droplets cited above [6,7]), it is relevant to consider cases in which the dimension of the system in the direction of the flow compares to the characteristic size of the structures that appear within the bed.

In the present article, we aim at the description of the destabilization of a thin immersed granular-layer submitted to an ascending liquid flow: We shall see that the destabilization of the layer generally leads to the appearance of a single localized instability. We consider that the layer is thin as long as the depth of the bed remains of the order of the characteristic size of the structure in the horizontal plane. Special attention has been paid to the design of a very simple experimental setup that makes it possible to impose a homogeneous hydraulic pressure-difference across a horizontal granular layer and to measure the resulting flow-rate. We determine the transition between the stable and unstable layer as well as the geometrical characteristics of the instability observed at the free surface. The observations are accounted by simple theoretical arguments.

\section{Experimental setup}

The experiment consists in measuring the liquid flow resulting from a pressure difference imposed to water between the upper and lower free-surfaces of an immersed layer of granular material. The experimental setup is made of three different parts (Fig. 1): One finds the sample in part A, whereas the overpressure $\Delta P$ is imposed by the system presented in part $B$. Finally, we use the tools sketched in part $\mathrm{C}$ to measure the resulting flow-rate.

A granular layer (thickness $h$ ) is confined by a vertical cylinder (total height $20 \mathrm{~cm}$, top of the experimental setup in part A) and lies at the bottom on a thin metallic grid (squares, $100 \mu \mathrm{m}$, thickness $\sim 0.2 \mathrm{~mm}$ ), which avoids the grains to fall down into the lower chamber. The inner diameter $d$ of the cylinder can be varied $(d=3.4$ or $9.1 \mathrm{~cm})$ and, in order to avoid the grains to slide at the side-walls and jetting to possibly occur preferably at the boundaries, glass spheres are glued onto the inner surface (diameter $400 \mu \mathrm{m}$, always larger than the typical grain-size). The whole system is filled with water and, when an ascending flow is imposed through the column of grains, the excess of water freely overflows at the top end of the vertical cylinder. Thus, in our experimental conditions, the height of liquid above the grains is constant (accuracy $1 \mathrm{~mm}$ ) and does not depend on the flow-rate, $v$. In addition, we image the free surface of the granular material along the vertical with the help of a digital camera.

The constant pressure difference $\Delta P$ is imposed to the system as follows: The lower chamber of part $\mathrm{A}$ is connected through a flexible pipe to the container of part B, which can be displaced up and down along the vertical (accuracy $1 \mathrm{~mm}$ ). Water from tap is injected at the bottom of the moveable container and the excess of water freely flows out to the sink through a hole situated above. The water level in the chamber does not depend on the liquid flow in the pipe connecting parts $\mathrm{A}$ and $\mathrm{B}$ and is known to within $1 \mathrm{~mm}$. We denote $\Delta z$ the height difference between the free surfaces of water in parts $\mathrm{A}$ and $\mathrm{B}$, so that the pressure difference between the bottom and the top of the granular column writes $\Delta P_{T}=$ $\rho g(\Delta z+h)$ where $\rho \simeq 10^{3} \mathrm{~kg} \mathrm{~m}^{-3}$ is the density of water and $g \simeq 10 \mathrm{~m} \mathrm{~s}^{-2}$, the acceleration due to gravity. The relation holds true as long as hydrostatics applies (i.e. as long as the water-flow does not lead to any significant pressure drop in the pipes and containers. We discuss this point in the next section 3. In the following, we denote $\Delta P \equiv \rho g \Delta z$ the contribution of the height difference $\Delta z$ to the overpressure $\Delta P_{T} ; \Delta P$ then excludes the equilibrium contribution $\rho g h$ of the hydrostatics, and according to this definition, the water tends to flow upwards when $\Delta P>0$. The maximum height difference is $\Delta z=10 \mathrm{~cm}$, corresponding to $\Delta P \simeq 10^{3} \mathrm{~Pa}$, whereas, taking into account the uncertainty in the liquid levels, the accuracy in $\Delta P$ is about $30 \mathrm{~Pa}$.

In order to characterize the ascending flow through the granular material, the water is collected at the top of the vertical tube (part A) and directed to a graduated cylinder (part C). Depending on the experimental conditions, we measure either the liquid volume $V$ (within $1 \mathrm{~mL}$ ) for a given time $\tau$ or the time $\tau$ (within $0.1 \mathrm{~s}$ ) for collecting a given volume $V$. Taking into account the surface area $\mathcal{A}=\pi(d / 2)^{2}$ of the cylinder cross-section, we can define the flow-rate, or Darcy velocity, $v \equiv V /(\mathcal{A} \tau)$, which is hence measured with an accuracy better than $1 \%$.

The granular material consists of spherical glass beads (sodosilicate glass, Matrasur Corp.). The samples are filtrated, so that the size dispersion is roughly $10 \%$ of the mean diameter $\phi$ of the grains (from $80 \mu \mathrm{m}$ to $400 \mu \mathrm{m}$ ).

\section{Experimental results and interpretation}

The experimental procedure is as follows: A given amount of granular material (up to $60 \mathrm{~mL}$, the corresponding height $h$ can be either larger or smaller than the inner diameter $d$ ) is placed in the vertical cylinder of part A. The sample is then stirred in order to remove any air bubble still trapped in the granular material. A pressure difference $\Delta P$ is imposed by lifting the container of part $B$ up to a chosen vertical position. After a short transient, the system reaches a steady regime 


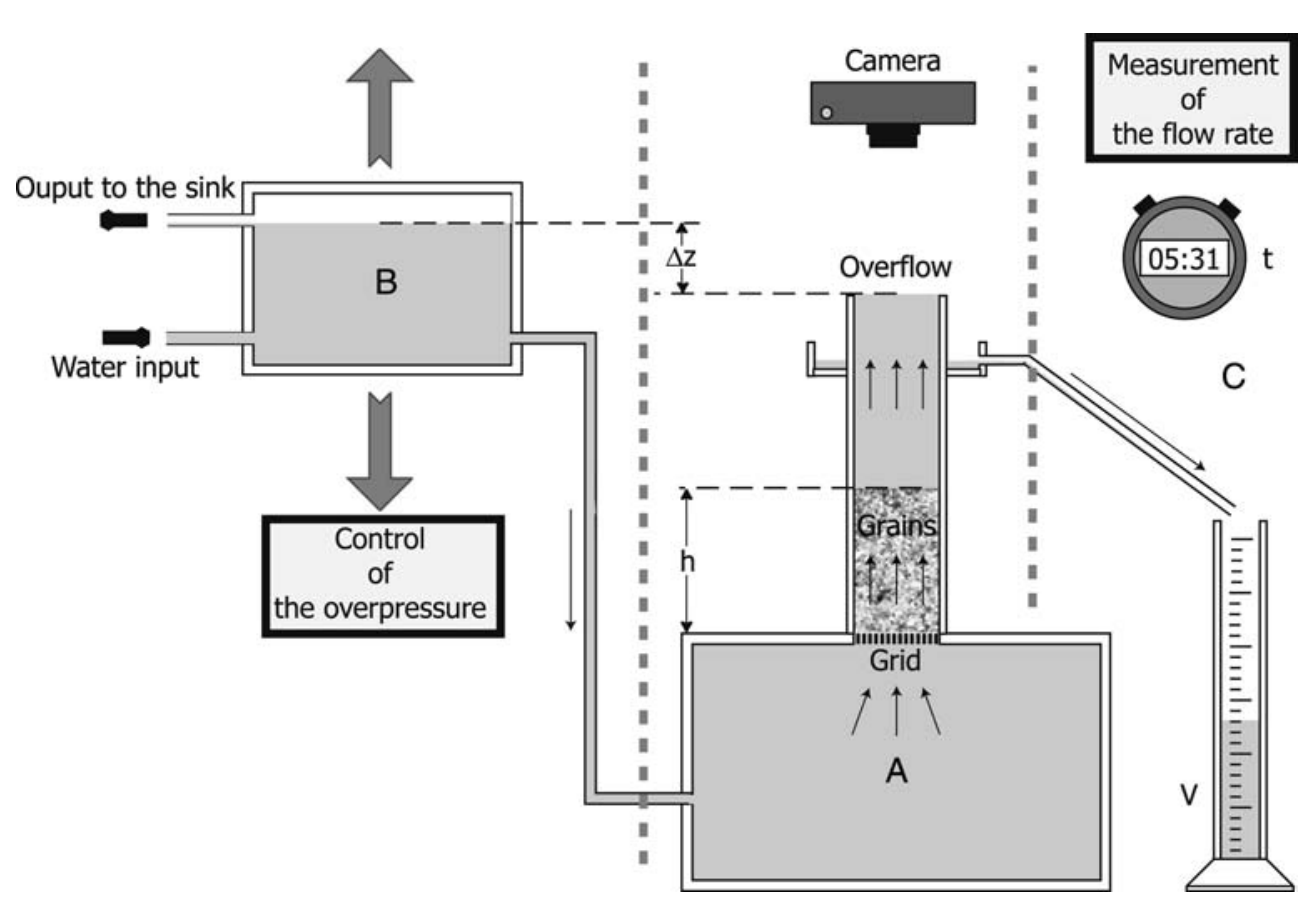

Fig. 1 Sketch of the experimental setup. The granular layer (part A, at the center) is subjected to an ascending water-flow imposed by changing the vertical position of the container sketched in part B (on the left-hand side). The resulting flow-rate is measured with the tools sketched in part $\mathrm{C}$ (on the right-hand side) [see text for details]

during which we measure the flow-rate $v$ and image the free surface.

\subsection{Typical response of the granular layer}

We report in figure 2 the typical behavior of $v$ in the steadystate as a function of $\Delta P$. One observes two linear regimes on both sides of a sharp transition which occurs at the critical pressure-difference $\Delta P^{*}$. For $\Delta P<\Delta P^{*}, v$ is proportional to $\Delta P$, and observation of the free surface from above proves that the granular material remains at rest in this regime. For $\Delta P>\Delta P^{*}, v$ depends linearly on $\Delta P$ according to $v=v^{*}+S\left(\Delta P-\Delta P^{*}\right)$ (the slope $S$ is larger than the slope measured below the transition). In this regime, some grains move; at the free surface of the granular layer, the motion of the grains is marked by the appearance of a small bump that moves freely and explores randomly the whole cross-section of the cylinder (Fig. 3).

In order to understand how the grains start moving at the transition, we reproduce the experiment in a $2 \mathrm{D}$-cell ${ }^{1}$. The experimental setup is similar to that presented in section 2 but the cylinder of part $\mathrm{A}$ is replaced by a thin cell consisting of two glass plates $\left(8 \times 10 \mathrm{~cm}^{2}\right)$ spaced by $1 \mathrm{~cm}$. The system is then imaged from the side. We observe that, at $\Delta P=\Delta P^{*}$, the whole granular layer dilates. A rapid subsequent destabilization of the "floating" granular-layer occurs; at the bottom of the cell appears locally a region in which the density of

\footnotetext{
${ }^{1}$ As we are only aiming at the qualitative description of the grains motion associated with the destabilization process, quantitative measurements have not been performed in the $2 \mathrm{D}$ setup.
}

the granular material is clearly less than the overall density. This region grows quickly in the vertical direction (Fig. 4), leading to the formation of a stable column of fluidized granular material which connects the bottom to the top of the granular layer. Simultaneously, the grains outside the fluidized region deposit back on the grid and stop moving. In the steady regime, the bump observed at the surface is a fountain of grains that ends the fluidized column.

In the following, we describe quantitatively the response of the granular layer to the liquid flow in the steady regime. Simple theoretical arguments account for the experimental observations.

\subsection{Pressure-drop in the pipe}

The experimental setup imposes a priori the pressure-difference $\Delta P$ across the granular layer. However, we point out that this is not true above the transition when $\Delta P$ departs significantly from $\Delta P^{*}$. Indeed, assuming a Poiseuille flow in the pipe (diameter $D \simeq 5 \mathrm{~mm}$ and length $L \simeq 50 \mathrm{~cm}$ typically) that connects the two containers of parts A and B, we estimate the slope $S=D^{4} /\left(32 \eta L d^{2}\right) \sim 3.810^{-5} \mathrm{~m}^{2} \mathrm{~s}$ $\mathrm{kg}^{-1}$ from the associated pressure drop. Experimentally, we measure $S=4.710^{-5} \mathrm{~m}^{2} \mathrm{~s} \mathrm{~kg}^{-1}$ (Section 3.1). Our estimate is of the same order of magnitude. Thus, at least for $\Delta P>\Delta P^{*}$, the flow-rate is mainly limited by the pressuredrop in the experimental setup, which thus rather imposes the flow-rate than a constant pressure-difference to the granular layer. In the following, we denote $\Delta P_{g}$, the pressure difference between the lower and the upper free surfaces of the 


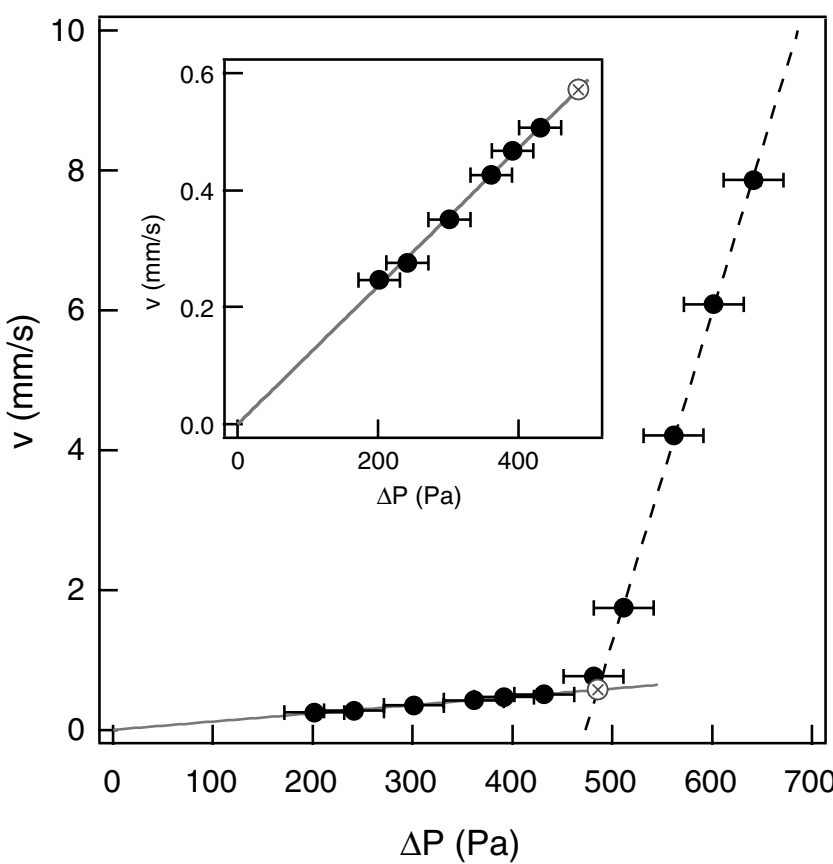

Fig. 2 Flow rate $\mathbf{v}$ vs. pressure difference $\Delta \boldsymbol{P}$. Symbols: experimental data. The response of the system to the imposed pressure difference $\Delta P$ exhibits two different linear regimes on both sides of a sharp transition [Cross: $\Delta P^{*}=485 \mathrm{~Pa}$, corresponding flow-rate $v^{*}=0.57 \mathrm{~mm} \mathrm{~s}^{-1}$ ]. Solid line: $\Delta P<\Delta P^{*}$, the flow-rate $v$ is proportional to the pressuredifference $\Delta P$; the system obeys Darcy law and we measure the intrinsic permeability $k=26 \mu \mathrm{m}^{2}$ (inset). Dashed line: $\Delta P>\Delta P^{*}, v$ increases linearly with $\Delta P$ with the slope $S=4.710^{-5} \mathrm{~m}^{2} \mathrm{~s} \mathrm{~kg}^{-1}(\phi=280 \mu \mathrm{m}$, $h=22 \mathrm{~mm}, d=3.4 \mathrm{~cm}$ )

granular material. In order to take into account the pressure drop in the setup, which cannot strictly be neglected even at small $\Delta P$, we write $\Delta P_{g}=\Delta P-v / S$. However, we note that the correction is small when $\Delta P<\Delta P^{*}$ [We measure $\left(\Delta P^{*}-\Delta P_{g}^{*}\right) / \Delta P^{*}<3 \%$ (Fig. 2)].

\subsection{Critical pressure difference $\Delta P_{g}^{*}$}

Destabilization of the granular layer occurs at the critical pressure difference $\Delta P^{*}$, associated with the critical flow-rate $v^{*}$. The critical values are accurately defined by the intersection of the two linear interpolations of $v(\Delta P)$ on both sides of the transition [Cross in Fig. 2]. Experimentally, the critical pressure difference $\Delta P_{g}^{*}$ does not depend on the diameter of the cylinder $d$ whereas it is found to be proportional to the thickness $h$ according to $\Delta P_{g}^{*}=\rho_{\text {exp }}^{*} g h$ with $\rho_{\text {exp }}^{*}=$ (0.80 \pm 0.01$) 10^{3} \mathrm{~kg} \mathrm{~m}^{-3}$ (Fig. 5).

In order to understand this experimental result, we estimate the pressure difference $\Delta P_{g}^{f l}$ associated with the fluidized granular column. We assume that, for $\Delta P_{g}=\Delta P_{g}^{*}$, the grains are only suspended by the liquid flow and do not move. From hydrostatics, $\Delta P_{g}^{f l}=c\left(\rho_{g}-\rho\right) g h$ where $\rho_{g}=$ $2.1910^{3} \mathrm{~kg} \mathrm{~m}^{-3}$ is the density of glass and $c$ the volume fraction of the grains. We measured, in addition, the density $c \rho_{g}=(1.53 \pm 0.05) 10^{3} \mathrm{~kg} \mathrm{~m}^{-3}$ of the granular material at

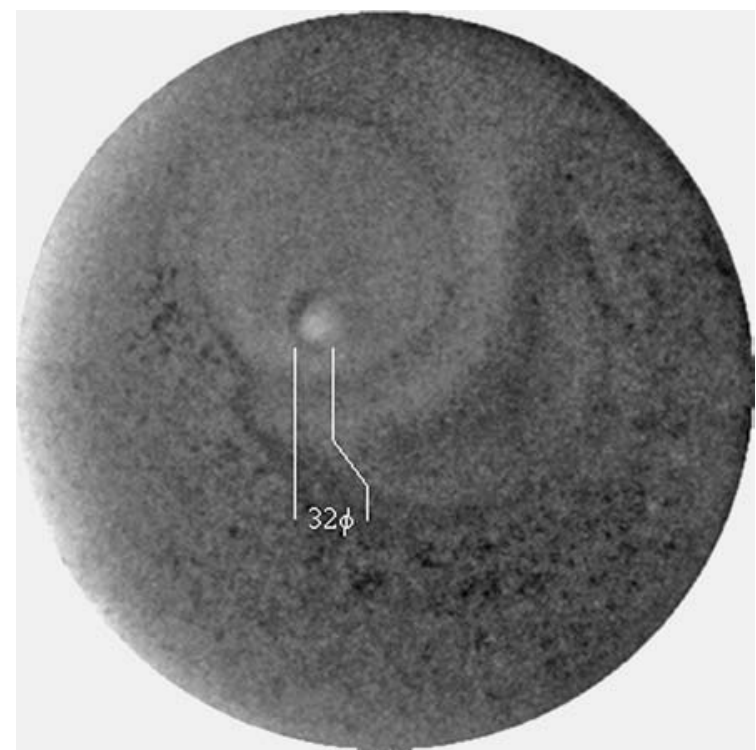

Fig. 3 Picture of the free-surface above the threshold. At the onset of the instability, one observes a small bump that moves freely and explores randomly the whole cross-section of the cylinder. The motion of the bump is marked by the unevenness of the free-surface $\left(v=1.2 \mathrm{~mm} \mathrm{~s}^{-1}\right.$, $\phi=142 \mu \mathrm{m}, h=16 \mathrm{~mm}, d=9.1 \mathrm{~cm})$
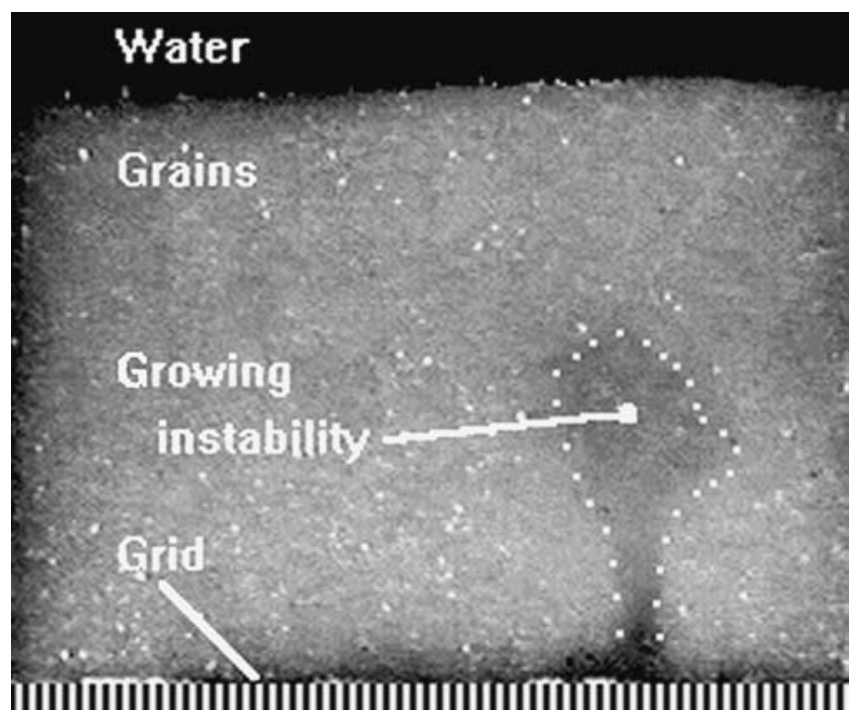

Fig. 4 Side-view of the fluidized granular column. At the onset of the instability, the whole granular layer slightly dilates and then destabilizes; from the side, one observes, at the bottom of the container, the nucleation of a localized region of smaller compacity (surrounded by the white dots) that quickly develops and reaches the upper free-surface $(\phi=360 \mu \mathrm{m}, h \simeq 55 \mathrm{~mm})$

rest, and deduced $c=(0.70 \pm 0.03)$. From these experimental values, we obtain $\rho_{\text {hyd }}^{*} \equiv c\left(\rho_{g}-\rho\right)=(0.83 \pm 0.03) 10^{3} \mathrm{~kg}$ $\mathrm{m}^{-3}$, which is only slightly larger than $\rho_{\text {exp }}^{*}$ [We note that, as $c$ is expected to be smaller when approaching the transition because of the slight dilation of the granular layer, the corresponding value of $\Delta P_{g}^{f l}$ is expected to slightly overestimate the real pressure difference]. The quantitative agree- 


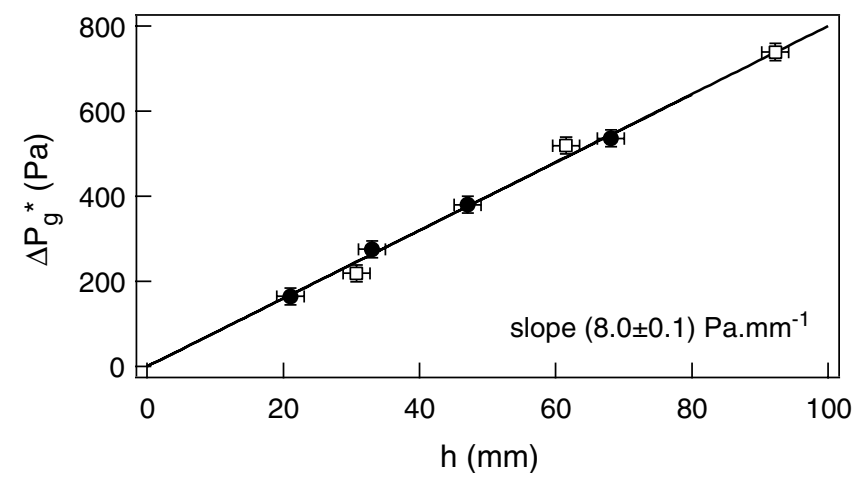

Fig. 5 Pressure-difference $\Delta \mathbf{P}_{\mathbf{g}}^{*}$ vs. thickness $\mathbf{h}$. Full symbols: $\phi=$ $360 \mu \mathrm{m}$; open symbols: $\phi=280 \mu \mathrm{m}$. The pressure difference measured at the transition is proportional to the thickness of the granular layer and does not depend on the bead diameter $\phi$

ment between the estimated, $\rho_{\text {hyd }}^{*}$, and measured, $\rho_{\text {exp }}^{*}$, values of $\rho^{*}$ shows that the instability occurs when the pressure force counterbalances the weight of the immersed granular layer, in agreement with former studies of fluidized beds [11-13]. The result also agrees with the observation that the granular layer is slightly lifted for $\Delta P=\Delta P^{*}$.

\subsection{The granular layer at rest $\left(\Delta P_{g}<\Delta P_{g}^{*}\right)$}

When $\Delta P_{g}<\Delta P_{g}^{*}$, we do not observe any motion of the grains. By changing the diameter $d$ of the tube and the thickness $h$ of the granular layer, we show that the flow-rate $v$ obeys Darcy law:

$v=\frac{k}{\eta} \frac{\Delta P_{g}}{h}$

where $\eta=10^{-3} \mathrm{~kg} \mathrm{~m}^{-1} \mathrm{~s}^{-1}$ denotes the dynamic viscosity of water ${ }^{2}$. We determine the intrinsic permeability, $k$ from the slope $v / \Delta P_{g}$. For instance (Fig. 2), we obtain $k=(26.6 \pm$ $0.2) \mu \mathrm{m}^{2}$ for $\phi=(280 \pm 30) \mu \mathrm{m}$. This value is in fairly good agreement with the empirical Kozeny-Carman relation [15]

$k=\frac{(1-c)^{3}}{c^{2}} \frac{\phi^{2}}{180}$

which leads to $k=(27 \pm 16) \mu \mathrm{m}^{2}$ for $c=(0.70 \pm 0.03)$.

We report, in addition, measurements of the flow rate $v^{*}$ at $\Delta P_{g}^{*}$. We know from section 3.3 that $\Delta P_{g}^{*}$ does not depend on the grain diameter $\phi$ and, from equation (2), we expect $k \propto \phi^{2}$ in the theory. In good agreement with this theoretical prediction, $v^{*}$ is observed to increase almost like $\phi^{2}$ (Fig. 6). Nevertheless, interpolation of the experimental results into a power law gives $v^{*} \propto \phi^{\alpha}$ with $\alpha=2.25 \pm 0.3$. The exponent $\alpha$ is found slightly larger than 2 . We measured, in addition, a slight increase of the volume fraction $c$ when the bead diameter $\phi$ is increased ( $c$ ranges from 0.67 to 0.72

2 Note that we did not check the dependance on the fluid viscosity $\eta$. By construction, the experimental setup does not make it possible to easily use another fluid than water from tap.

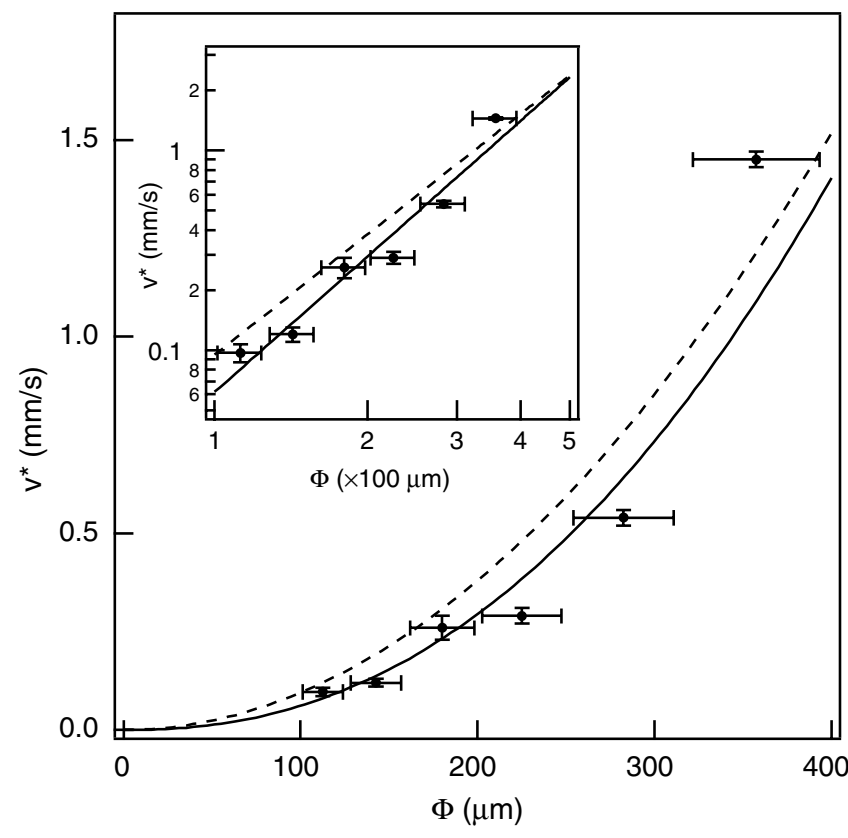

Fig. 6 Critical flow-rate $\mathbf{v}^{*}$ vs. bead diameter $\phi$. Inset: log-log plot. Symbols: experimental data. Solid line: $v^{*} \propto \phi^{\alpha}$ with $\alpha=2.25$. Dashed line: $v^{*} \propto \phi^{2}$

for $\phi=90$ to $715 \mu \mathrm{m}$ ), which is likely to explain that the experimental value of $\alpha$ is larger than 2 . The polydispersity of the samples may be responsible for the measured changes in both the compacity $c$ and the intrinsic permeability $k$. Note that there exists, in the literature [11-13], several relations which can account for the variations of $k$ for polydisperse samples and irregular grains. The lack of precise characterization of our samples does not allow us to discuss further this point. However, our experimental results prove that, in this regime, the response of the granular layer is that of a usual porous material.

\subsection{The granular layer above the transition $\left(\Delta P_{g}>\Delta P_{g}^{*}\right)$}

For $\Delta P_{g}>\Delta P_{g}^{*}$, the grains move and one observes from the side a column of fluidized granular material, which ends at the free surface by a localized fountain. Seen from above, the fountain exhibits a well-defined typical diameter, which we discuss in the next section 3.5.1.

\subsubsection{Typical size of the localized instability}

Whereas the height of the fountain above the free surface of the granular layer largely fluctuates in time, its diameter $\Theta$ in the horizontal plane is almost constant, independent of both the pressure difference $\Delta P$ and the height $h$. We point out that the random motion of the fountain at the free surface of the layer attests that its geometry is also not governed by the inner diameter $d$ of the container. We measure $\Theta \simeq 32 \phi$, where $\phi$ is the typical diameter of the grains. The result holds true even when the thickness $h$ is less than $\Theta$ (we performed 
experiments with grains having diameters between 315 and $400 \mu \mathrm{m}$ and heights $h=3 \mathrm{~mm}$ and $6 \mathrm{~mm}$, corresponding respectively to 8 and 16 layers of grains).

We can recover the scaling law $\Theta \propto \phi$ by writing the total volume flow-rate through the granular layer $Q_{T}$ in the form $Q_{T}=Q_{C}+Q_{L}$ where $Q_{C}$ denotes the flow-rate in the fluidized column and $Q_{L}$ the flow-rate in the remaining part of the granular layer which is at rest. Assuming that the pressure difference across the granular layer $\Delta P_{g}$ is homogeneous in the whole system and that the stream lines remain vertical, we can write $Q_{C}=\frac{\pi}{4} \Theta^{2} J_{C}$ and $Q_{L}=\frac{\pi}{4}\left(d^{2}-\right.$ $\left.\Theta^{2}\right) J_{L}$ where $J_{C}$ and $J_{L}$ denote the currents in the column and in the layer at rest respectively (Fig. 7). From equation (1), we get $J_{L}=(k / \eta)\left(\Delta P_{g} / h\right)$. Assuming a Poiseuille flow in the fluidized column, we write $J_{C}=\left(\Theta^{2} / 32 \eta_{f}\right)\left(\Delta s P_{g} / h\right)$ where $\eta_{f}$ stands for the viscosity of the fluidized material. With these assumptions,

$Q_{T}=\left[\frac{\pi}{4}\left(d^{2}-\Theta^{2}\right) \frac{k}{\eta}+\frac{\pi}{128 \eta_{f}} \Theta^{4}\right] \frac{\Delta P_{g}}{h}$

and reaches a maximum value for $\Theta^{2}=16 k\left(\eta_{f} / \eta\right)$. From equation (2), which shows that $k \propto \phi^{2}$, we recover $\Theta \propto \phi$, independent of $h$ and $\Delta P_{g}$.

We do not expect any quantitative agreement between our oversimplified model and the experiment. Indeed, in order to obtain $\Theta=32 \phi$, one must take $\eta_{f} / \eta \sim 2 \times 10^{5}$. From equation (3), we then expect the variation of the flow-rate associated with the instability to be $\delta Q_{T} / Q_{T} \sim 4 \%$. Thus, our simple argument fails to account for the large variation of the slope $\mathrm{d} v / \mathrm{d}(\Delta P)$ at the transition. The phenomenon is likely to be explained by a drastic change in the compacity $c$ of the whole granular layer for $\Delta P_{g}>\Delta P_{g}^{*}$ in agreement with the observation that, at the transition, the whole granular layer is slightly lifted by the flow before the localized instability appears. Nevertheless, we note that there exist several theoretical or empirical models that relate the viscosity $\eta_{f}$ to the corresponding compacity $c_{f}$ of the fluidized material [16]. Whatever the model, such large value of $\eta_{f} / \eta$ is explained by $c_{f}$ only slightly smaller than $c$. For instance, by using Mooney's relation [17], which states that:

$$
\frac{\eta_{f}}{\eta}=\exp \left(\frac{2.5 c_{f}}{1-c_{f} / c}\right)
$$

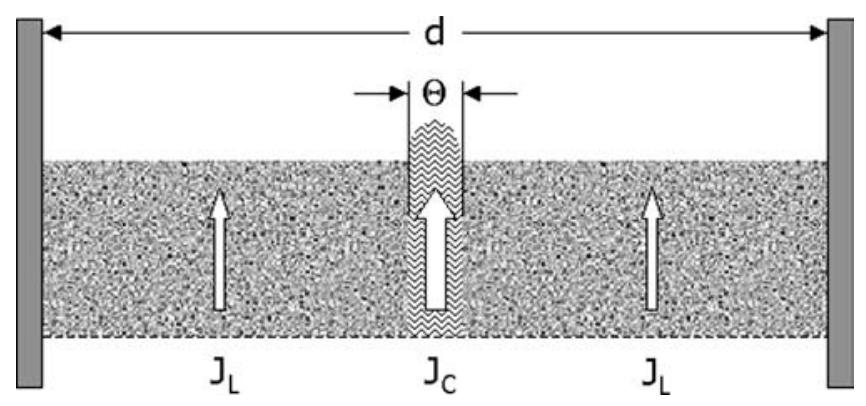

Fig. 7 Sketch of the theoretical situation. We assume that the grains only move in a cylinder of diameter $\Theta$, whereas they remain at rest elsewhere in the granular layer we get $c_{f} \sim 0.61$, roughly $10 \%$ smaller than $c$. The compacity of the fluidized material that flows would be only $10 \%$ less than that of the material at rest.

Thus, in spite of numerous assumptions, our simple model makes possible to recover that the typical diameter $\Theta$ of the localized instability scales like the typical grain size $\phi$ and is independent of both the thickness $h$ of the granular layer and the pressure difference $\Delta P_{g}$.

\subsubsection{Flow rate $v\left(\Delta P_{g}>\Delta P_{g}^{*}\right)$.}

Above the transition, neglecting the pressure drop due to the granular layer, we can write $\Delta P=\Delta P_{g}^{*}+v / S$ where $\Delta P_{g}^{*}$ stands for the contribution of hydrostatics in the fluidized granular column and $v / S$ to the pressure drop in the experimental setup. As a consequence, $v$ can be rewritten in the form $v=S\left(\Delta P-\Delta P^{*}\right)+v^{*}$ provided that $v^{*}=S\left(\Delta P^{*}-\Delta P_{g}^{*}\right)$. Thus, $v^{*}$ corresponds to the water flow-rate associated with the pressure-difference necessary for counterbalancing the weight of the granular layer. This explains the offset in the $v(\Delta P)$ characteristics $[v(\Delta P)$ reaches the $\Delta P$-axis at a finite pressure difference].

\section{Conclusion}

An thin immersed granular layer, subjected to an ascending liquid-flow, destabilizes when the pressure force counterbalances the weight of the granular material. Instead of being homogeneously lifted by the liquid flow, the granular material locally destabilizes: a channel of less dense material nucleates at the bottom and quickly reaches the upper free surface. The resulting steady situation consists of a column

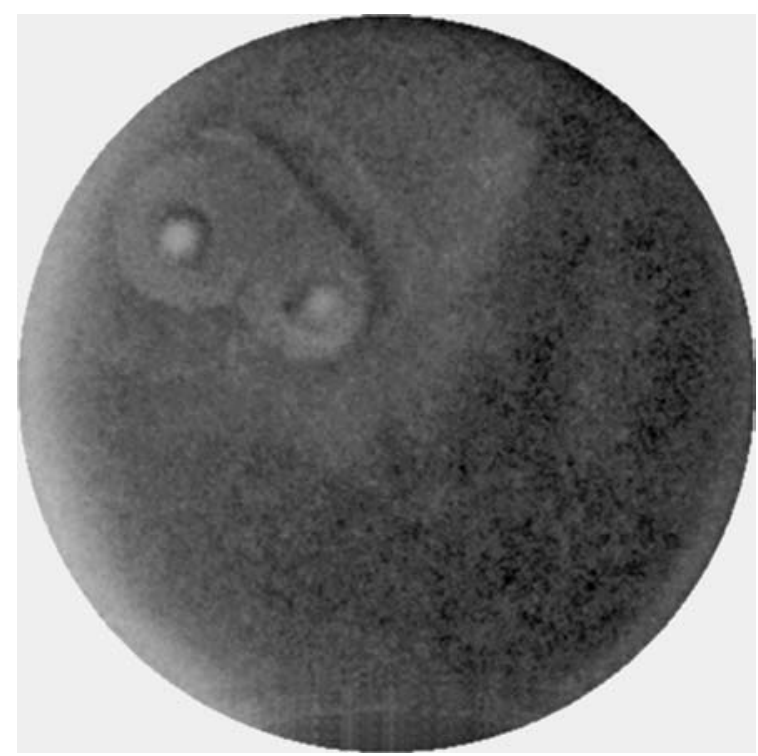

Fig. 8 Second instability at the free surface. Whereas the diameter $\Theta$ does not depend on the flow-rate, further increase of $\Delta P$ can lead to the nucleation of a second fountain which also moves freely at the free surface $(\phi=142 \mu \mathrm{m}, h=16 \mathrm{~mm}, d=9.1 \mathrm{~cm})$ 
of fluidized material that connects the bottom to the top of the granular layer. Observation of the free surface exhibits a small fountain of grains, which typical diameter is of about 32 times the grains diameter: the result can be, at least qualitatively accounted, by a simple theoretical argument based on the optimization of the flow-rate at a given imposed pressure difference.

Nevertheless, even if we obtained reliable informations from these preliminary experiments, the setup presents several flaws which avoid exploring further the phenomenon. For instance, the results demonstrate that the experimental setup fails in imposing the pressure difference across the granular layer. Increasing further $\Delta P$ above $\Delta P^{*}$, we observed the appearance of a second fountain at the free surface of the granular layer (Fig. 8). The fact that this second instability occurs at larger $\Delta P$ may be explained by a pressure drop in the tubes resulting from the first instability at $\Delta P^{*}$, which is associated with a noticeable increase in the flow-rate. In order to better understand the phenomenon, we are planning to impose the flow-rate and measure the resulting pressure at the bottom of the layer. In addition, increasing the diameter of the container will make it possible to analyze the free motion of the fountains at the free surface and the interaction between them. This work will be extended to the case of binary mixtures of granular materials in order to understand the role of the polydispersity. These experiments are in preparation.

The authors thank H. Gayvallet and F. Melo for fruitful discussions.

\section{References}

1. Dudukovic, M.P., Larachi, F., Mills, P.L.: Chem. Engng. Sci. 54, 1975 (1999); J.B. Joshi, Chem. Engng. Sci. 56, 5893 (2001)

2. Bagnold, R.A.: Motion of waves in shallow water. Interaction between waves and sand bottoms. In: The physics of sediment transport by wind and water C.R. Throne, R.C. MacAthur, J.B. Bradley (eds.), American Society of Civil Engineers, New-York, 1988

3. Persson, B.N.J.: In: Solid friction. Springer, New-York, 1998

4. Komatsu, T.S., Inagaki, S., Nakagawa, N., Nasuno, S.: Phys. Rev. Lett. 86, 1757-1760 (2001)

5. Geminard, J.-C., Losert, W., Gollub, J.P.: Phys. Rev. E 59, 5881 (1999)

6. Duran, J.: Comptes Rendus Physique 3, 217-227 (2002)

7. Caballero, L., Melo, F.: Phys. Rev. Lett. 93, 258001 (2004)

8. Derec, C., Senis, D., Talini, L., Allain, C.: Phys. Rev. E. 67, 062401 (2003)

9. Darcy, H.: In: Les fontaines publiques de la ville de Dijon. Dalmont, Paris, 1856

10. Wong, R.C.K.: Can. Geotech. J. 40, 95-106 (2003)

11. Geldart, D.: Gas Fluidisation Technology. John Wiley \& Sons, 1986

12. Coulson, J.M., Richardson, J.F.: Chemical Engineering, Vol. 2, $4^{\text {th }}$ edn., 1991

13. Kunii, D., Levenspiel, O.: Fluidisation Engineering, $2^{\text {nd }}$ edn., 1991

14. Mathur, K.B., Epstein, N.: Spouted Beds. Academic Press, 1974

15. Carman, P.C.: In: Flow of gases through porous media. Butterworths, London, 1956

16. Cheng, N.-C., Law, A.W.K.: Powder Tech. 129, 156-160 (2003)

17. Mooney, J.: J. Colloid Sci. 6, 162-170 (1951) 